\title{
Vilosite Placentária e sua Relação com Intercorrências Fetais e Maternas
}

\author{
Placental Villitis and its Relationship with Fetal and Maternal Outcome
}

\begin{abstract}
Eumenia Costa da Cunha Castro, Ana Karina Marques Salge, Fabiana Jorge Bueno Galdino, Mara Lúcia Fonseca Ferraz, Marlene Antônia dos Reis, Rosana Rosa Miranda Corrêa, Vicente de Paula Antunes Teixeira
\end{abstract}

\begin{abstract}
RESUM0
Objetivo: a vilosite placentária tem sido associada a infecção perinatal, embora uma porcentagem permaneça de etiologia desconhecida. O objetivo deste estudo foi realizar análise morfológica das vilosites, com caracterização imuno-histoquímica, e relacioná-la com intercorrências maternas e fetais.

Métodos: foram incluidas 128 placentas. Foi realizada a análise macroscópica e todos os fragmentos coletados foram analisados microscopicamente pelo método da hematoxilinaeosina. As vilosites foram classificadas de acordo com a intensidade do processo inflamatório em vilosite discreta, moderada e acentuada. Para a pesquisa do agente etiológico foi realizada imuno-histoquímica utilizando anticorpos monoclonais anti-Toxoplasma gondii $e$ anti-Cytomegalovirus. Para avaliação do fenótipo das células do infiltrado inflamatório das vilosidades foram utilizados os seguintes anticorpos: anti-CD68, anti-CD57, CD3 e CD20. Foram analisadas as seguintes variáveis: idade materna e gestacional, peso fetal e placentário e intercorrências maternas e fetais. Na comparação entre dois grupos utilizamos o teste de Mann-Whitney e as proporções foram comparadas por meio do teste do $\chi^{2}$. As diferenças observadas foram consideradas significativas quando $p<0,05$ (5\%).

Resultados: a vilosite foi identificada em 11,7\% das placentas. Em $40 \%$ dos casos de vilosite as crianças foram natimortas ( $p=0,003)$. Foi encontrado um caso de toxoplasmose congênita e os demais foram classificados como vilosite de etiologia desconhecida.

Conclusões: a intensidade do processo inflamatório placentário apresentou relação com a gravidade do acometimento fetal. As células inflamatórias que compõem a vilosite são macrófagos em sua maioria. Entretanto, não foi possível identificar os agentes etiológicos responsáveis pela processo inflamatório.
\end{abstract}

PALAVRAS-CHAVE: Placenta. Vilosite. Gravidez: infecções. Toxoplasmose.

\section{Introdução}

Devido à dependência da ligação entre a placenta e o feto, a alteração da função placentária

Disciplina de Patologia Geral, Departamento de Ciências Biológicas da Faculdade de Medicina do Triângulo Mineiro. Correspondência:

Eumenia Costa da Cunha Castro

Disciplina de Patologia Geral - Departamento de Ciências Biológicas

Faculdade de Medicina do Triângulo Mineiro

Avenida Frei Paulino n 30 - Bairro Abadia

38025-180 - Uberaba - MG

Telefone: (34) 3318-5428 - Fax: (34) 3318-5462

email: eumenia.pat@dcb.fmtm.br

Apoio financeiro: FAPEMIG, FUNEPU (Fundação de Ensino e Pesquisa de Uberaba) e CNPq. pode predizer diferentes padrões de resultados de gestações e estabelecer a evolução de lesão fetal ${ }^{1}$. Além de ser importante instrumento para identificação da causa de morte, o estudo da placenta pode muitas vezes indicar o prognóstico de sucesso de gestações futuras ${ }^{2}$.

Ầs infecções congênitas como a sífilis ${ }^{3}$, toxoplasmose, síndrome da imunodeficiência adquirida $^{4}$, varicela, rubéola, herpes, citomegalovirose ${ }^{5}$ e as causadas por outros agentes infecciosos como por exemplo o Trypanosoma cruzi, entre outros ${ }^{6}$, também estão associados padrões de lesões placentárias que levam à suspeita diagnóstica. $\mathrm{O}$ encontro do agente etiológico com o uso de técnicas de histoquímica, biologia molecular e imunohistoquímica confirma o diagnóstico ${ }^{7}$. 
As vilosites crônicas foram mencionadas primeiramente como um dos sinais de insuficiência placentária $^{8}$. Alguns autores descreveram a entidade em detalhes e, após a descrição, esta entidade tem sido motivo de diversos estudos ao longo dos $\operatorname{anos}^{9}$. A vilosite se caracteriza pelo infiltrado de leucócitos focal ou multifocal no estroma das vilosidades coriônicas, usualmente associada com necrose fibrinóide do sinciciotrofoblasto. As vilosites crônicas são encontradas em aproximadamente $10 \%$ das placentas e estão associadas com o aumento do risco da morbidade e mortalidade neonatal ${ }^{10}$. Entre as crianças que apresentaram intercorrências no período perinatal, as placentas podem apresentar vilosite crônica em até 75\% dos casos $^{11}$. As principais intercorrências relacionadas às vilosites são: prematuridade, recém-nascidos pequenos para a idade gestacional ${ }^{12,13}$, malformações congênitas, abortamento de repetição e restrição do crescimento intra-uterino ${ }^{14}$.

As vilosites podem ser de etiopatogenia conhecida, quando estão relacionadas com agente infeccioso conhecido ${ }^{10}$, ou ainda podem aparecer sem que se possa encontrar nenhum agente responsável, sendo estas chamadas "vilosites de etiologia desconhecida"15,16. A etiopatogênese tanto das vilosites de etiologia conhecida como as de origem desconhecida não está bem estabelecida ${ }^{17}$. Além dos agentes etiológicos mais comuns das infecções congênitas, entram no diagnóstico diferencial das vilosites de etiologia desconhecida as manifestações imunes da mãe contra o feto, encontradas nos abortos de repetição ${ }^{18}$.

O objetivo do nosso trabalho foi o estudo morfológico sistemático das placentas de partos realizados no Hospital Escola da Faculdade de Medicina do Triângulo Mineiro, para diagnóstico morfológico das vilosites, sua caracterização imuno-histoquímica com o uso de marcadores imunológicos e sua possível relação com intercorrências maternas e fetais.

\section{Material e Métodos}

Foi realizado estudo retrospectivo transversal. Foram incluídas 128 placentas, coletadas de partos consecutivos realizados no Hospital Escola da Faculdade de Medicina do Triângulo Mineiro, Uberaba, Minas Gerais (HE-FMTM), durante os anos de 1998 e 1999. Não foi excluída nenhuma placenta.

Fizemos a revisão do prontuário de todas as pacientes que tiveram as placentas examinadas, com o objetivo de colher as informações relativas à idade, à cor e à paridade materna, ao sexo, à idade gestacional e ao peso da criança, a gestação única ou gemelar e a intercorrências fetais e maternas.
Após a análise macroscópica foram colhidos dez fragmentos da placenta: um fragmento da membrana livre corioamniótica, um fragmento do parênquima placentário na inserção do cordão umbilical, dois fragmentos do cordão umbilical e seis fragmentos coletados de forma randomizada do centro do disco placentário. Todos os fragmentos foram analisados morfologicamente pelo método da hematoxilina-eosina. As placentas foram divididas em dois grupos: com vilosite e sem vilosite. No grupo de placentas com vilosite foram classificadas as placentas que apresentavam infiltrado mononuclear no córion vilositário.

As vilosites foram classificadas de acordo com a intensidade do processo inflamatório em vilosite discreta, quando encontramos não mais que um foco de inflamação, em aumento de 5 vezes, e no total do corte menos de $25 \%$ dos campos examinados; vilosite moderada, quando encontramos freqüentemente lesões focais em mais de $25 \%$ dos campos examinados; e vilosite acentuada, quando o acometimento das vilosidades era generalizado, sendo o processo inflamatório difuso por quase todas as vilosidades ${ }^{14,19}$.

Para a pesquisa do possivel agente etiológico foi realizada a reação imuno-histoquímica utilizando anticorpos monoclonais ou policlonais antiToxoplasma gondii e anti-Citomegalovirus. Para avaliação do fenótipo das células mononucleares encontradas no infiltrado das vilosidades foram utilizados os seguintes anticorpos: anti-CD68 para marcação de macrófagos, anti-CD57 para marcação de células natural killer, CD3 para linfócitos T e CD20 para linfócitos B. Para o Treponema pallidum foi realizada a coloração de Warthin-Starry.

Após desparafinização, os cortes histológicos foram lavados em tampão Tris- $\mathrm{NaCl}$ 0,05, pH 7,6, por 15 minutos à temperatura ambiente e tratados com metanol e $\mathrm{H}_{2} \mathrm{O}_{2}$, para bloqueio da peroxidase endógena dos tecidos. A seguir, os cortes foram incubados em solução contendo o anticorpo primário, albumina sérica bovina a 1\% e tampão Tris-NaCl, durante duas horas à temperatura ambiente e lavados com TRIS- $\mathrm{NaCl}$ da mesma maneira que anteriormente. Após a incubação com o anticorpo primário as lâminas foram incubadas com os anticorpos secundários por mais 90 minutos. Os anticorpos secundários utilizados foram conjugados com peroxidase ou biotina $\left(\mathrm{DAKO}^{\circledR}\right)$. A revelação foi realizada com diaminobenzidina $\left(\mathrm{DAB}^{\circledR}\right)$ ou por meio do sistema avidinabiotina (Sistema Vectastain Elite ABC-Vector ${ }^{\circledR}$ ), dependendo do anticorpo secundário. Logo após os cortes foram lavados em água destilada, desidratados e montados com Entelan ${ }^{\circledR}$.

Para a análise estatística foi elaborado um banco de dados eletrônico no Microsoft Excel ${ }^{\circledR}$. As informações foram analisadas utilizando-se o pro- 
grama eletrônico Sigma Stat ${ }^{\circledR}$ versão 2.0. As variáveis foram testadas para verificar se tinham distribuição normal, pelo teste de Kolmogorov-Smirnov. Na comparação entre dois grupos utilizamos o teste de Mann-Whitney, para distribuição não normal. As proporções foram comparadas por meio do teste do $\chi^{2}$. As diferenças observadas foram consideradas significativas quando a probabilidade de rejeição da hipótese de nulidade foi menor que 0,05 (5\%).

O estudo foi aprovado pelo Comitê de Ética em Pesquisa da Faculdade de Medicina do Triângulo Mineiro (FMTM), protocolo 011 CEP/FMTM.

\section{Resultados}

Das 128 placentas, quinze $(11,7 \%)$ apresentaram o diagnóstico de vilosite. Os dados da Tabela 1 representam os parâmetros analisados nos casos de vilosite. A idade das mães nos casos em que foi diagnosticada vilosite foi significantemente maior do que nos casos sem alteração patológica placentária $(\mathrm{p}=0,012)$ (Tabela 1$)$.

Tabela 1 - Características dos casos com vilosite comparados com os casos nos quais não se encontrou alteração patológica nas placentas.

\begin{tabular}{|c|c|c|c|c|c|c|c|c|}
\hline & \multicolumn{4}{|c|}{ Sem alteração patológica placentária } & \multicolumn{4}{|c|}{ Com vilosite } \\
\hline & $\overline{\mathrm{x}} \pm \mathrm{DP}$ & M & $\operatorname{Max}$ & Min & $\bar{x} \pm \mathrm{DP}$ & M & Max & Min \\
\hline $\mathrm{IM}(\mathrm{anos})^{*}$ & $23,8 \pm 5,7$ & 19,0 & 37,0 & 14,0 & $27,4 \pm 7,0$ & 25,0 & 40,0 & 12,0 \\
\hline$I G(s)^{\star \star}$ & $39,3 \pm 1,6$ & 39,5 & 43,0 & 34,0 & $33,6 \pm 8,2$ & 38,0 & 42,0 & 18,0 \\
\hline$P F(g)$ & $3144,6 \pm 526,9$ & 3125,0 & 4870,0 & 2170,0 & $2253,1 \pm 1278,3$ & 2880,0 & 3700,0 & 270,0 \\
\hline PP (g) & $444,0 \pm 105,8$ & 450,0 & 750,0 & 240,0 & $326,7 \pm 114,1$ & 350,0 & 510,0 & 150,0 \\
\hline
\end{tabular}

O peso das crianças a termo em cujas placentas foi feito o diagnóstico de vilosite foi menor do que daquelas crianças em que as placentas foram consideradas normais; no entanto, esta diferença não foi estatisticamente significante. Quando analisamos o subgrupo das crianças prematuras com vilosite, o peso fetal foi estatisticamente menor do que nas crianças prematuras sem vilosite $(\mathrm{p}=0,014)$.

Quanto à intensidade do processo inflamatório, seis $(40,0 \%)$ casos foram classificados como vilosite discreta, seis (40\%) como vilosite moderada e três (20\%) como vilosite acentuada (Tabela 2).

Tabela 2 - Distribuição das intercorrências maternais e fetais e a intensidade do infiltrado inflamatório entre os casos em que foi diagnosticada vilosite nas placentas.

\begin{tabular}{cccc}
\hline Caso & Intercorrência materna & Intercorrência fetal & Intensidade da inflamação \\
\hline 1 & Parto prematuro & Gemelares & Discreta \\
2 & Normal & Gemelares & Doderada \\
3 & Parto prematuro & Natimorto & Moderada \\
4 & Parto prematuro & Sofrimento fetal & Acentuada \\
5 & Cetoacidose diabética & Natimorto & Moderada \\
6 & Incompetência istmo cervical & Natimorto & Acentuada \\
7 & Toxoplasmose congênita & Natimorto & Moderada \\
8 & Normal & Natimorto & Discreta \\
9 & Normal & Infecção perinatal & Discreta \\
10 & Normal & Infecção perinatal & Discreta \\
11 & Circular de cordão & Natimorto & Moderada \\
12 & DHEG & Gemelar & Discreta \\
13 & DHEG & Gemelar & Moderada \\
14 & Epilepsia & Sofrimento fetal & Acentuada \\
\hline
\end{tabular}

*Vilosite vs Natimorto: $\chi^{2}=9,073 ; p=0,003$. 
Dos casos com vilosite analisados, dez (66,6\%) apresentaram células CD68+ no interior das vilosidades (Figura 1D) e cinco $(33,3 \%)$ apresentaram marcação CD68+ também no espaço intervilositário. Em cinco $(33,3 \%)$ casos a marcação foi negativa para CD68 (Figura 1B). Em um caso $(7,7 \%)$ encontramos células positivas para CD3 em algumas vilosidades afetadas. Os demais marcadores utilizados, CD57 e CD20, foram negativos em todos os casos analisados.

A avaliação imunohistoquímica para os agentes infecciosos foi positiva em um $(6,7 \%)$ caso para toxoplasmose (Figura 1E). Os demais casos $(93,3 \%)$ foram negativos para todos os agentes testados, sendo classificados como vilosite de etiologia desconhecida.
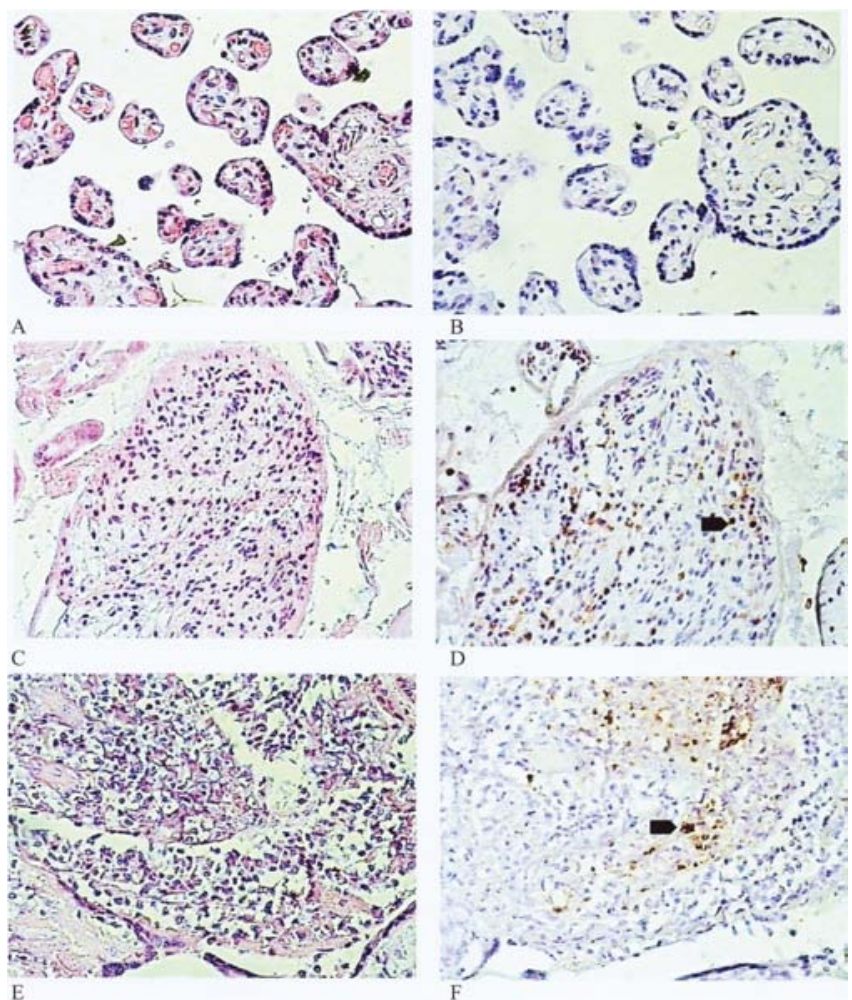

Figura 1 - Morfologia das vilosidades placentárias após análise imuno-histoquímica. As vilosidades sem alteração (A) não apresentam macrófagos CD68+ no mesoderma (B). As células que compõem as vilosites (C) são na sua maioria macrófagos CD68+ (seta) (D). Em um dos casos de vilosite (E), encontramos positividade para o Toxoplasma gondii (seta) (F). Todas as figuras da esquerda foram coradas pelo $\mathrm{HE}$ e as da direita pelo método peroxidade antiperoxidase com anticorpos anti-CD68+ (D) e anti-Toxoplasma gondii (F) (X640)

\section{Discussão}

A vilosite placentária foi encontrada em $11,7 \%$ das placentas analisadas. Na literatura a freqüência de vilosite varia de 2,5 a $10 \%$ em placentas examinadas rotineiramente e está asso- ciada com o aumento do risco da morbidade e mortalidade neonatal, chegando esta relação a 100\% em alguns trabalhos ${ }^{10}$.

Em 86,7\% dos nossos casos encontramos alterações fetais ou maternas associadas à vilosite placentária. Somente em um dos casos analisados conseguimos definir o agente etiológico com o uso de imuno-histoquímica. Estes dados estão de acordo com a constatação de que, na maioria dos casos de vilosite placentária, não se consegue determinar o agente etiológico, sendo então classificadas como vilosite de etiologia desconhecida ${ }^{20}$

Em nossa casuística as intercorrências maternas e fetais com vilosite foram desde distúrbios metabólicos, como diabetes, até alterações infecciosas, como toxoplasmose congênita, mas o diagnóstico de infecção foi feito em somente 13,3\% dos casos. Estes dados estão de acordo com relatos de casos de vilosite de etiologia desconhecida relacionados a abortamentos de repetição, em que os conceptos raramente apresentaram sinais de infecção, mas foram sempre afetados por outros processos patológicos ${ }^{20}$. No entanto, houve um grupo de crianças com vilosite no qual, além de não termos conseguido identificar um agente infeccioso, a mãe não apresentou nenhuma intercorrência no período gestacional que pudesse explicar o aparecimento de vilosite. O que havia em comum entre estas crianças é que as mães tinham idade maior do que as mães em que encontramos outras alterações placentárias que não vilosite. A vilosite tem sido correlacionada com gestações sucessivas ou multiparidade e ainda com abortamentos de repetição ${ }^{19}$. A multiparidade comumente é encontrada em mulheres mais velhas e a idade materna elevada é um fator de risco para abortamento espontâneo ${ }^{21}$.

As vilosites de etiologia desconhecida têm sido vistas associadas com intercorrências fetais, como prematuridade, recém-nascidos pequenos para a idade gestacional, malformações congênitas, abortamento de repetição e restrição do crescimento intra-uterino ${ }^{13,22}$. Em nossa casuística encontramos relação estatisticamente significante entre natimortalidade e vilosite. A intensidade do processo inflamatório tem sido correlacionada com a freqüência e a gravidade das alterações fetai ${ }^{14}$. A natimortalidade foi tanto maior quanto maior a intensidade do processo inflamatório, sendo que entre os prematuros que apresentaram vilosite acentuada a mortalidade foi de $100 \%$. Nos casos em que não encontramos intercorrência fetal associada à vilosite, a intensidade do processo foi discreta em $80 \%$ dos casos. Estes dados demonstram que a intensidade do processo inflama- 
tório se relacionaria diretamente com o aumento da freqüência de intercorrências fetais.

A natureza das células que compõem o infiltrado inflamatório nos casos de vilosites são os macrófagos CD68+, histiócitos e linfócitos T CD3+, todos de provável origem materna ${ }^{23}$. Em nosso estudo as células que constituem as vilosite foram macrófagos CD68+ na maioria dos casos e em um caso encontramos linfócitos T compondo o infiltrado. No entanto, no interior das vilosidades acometidas, encontramos outras células que não macrófagos com fenótipo CD68+, demonstrando que outras células podem também fazer parte das vilosites, como demonstrado por outros estudos. Ao contrário do descrito na literatura, em somente um caso encontramos células positivas para anticorpo contra linfócitos $\mathrm{T}^{24}$. Concordamos com outros autores em que o aspecto e a natureza das células das vilosites, nos casos em que não conseguimos identificar o agente etiológico e naqueles em que conseguimos, não apresentam diferença ${ }^{10}$.

Em nosso estudo, o encontro das vilosites só foi associado à presença de agente etiológico em um caso, nos demais não encontramos agentes infecciosos, nos levando à mesma conclusão descrita na literatura de que na maior parte das vilosites placentárias não é possível a identificação de agentes infecciosos, sendo classificadas como "vilosites de etiologia desconhecida". No entanto, a intensidade do processo está relacionada ao aumento de intercorrências fetais, principalmente a natimortalidade. Quanto à natureza das células que constituem as vilosites, em nosso estudo encontramos em sua maioria macrófagos CD68+. Mas na maior parte das vilosidades afetadas a celularidade estava aumentada às custas de mononucleares e somente parte das células marcaram positivamente para CD68 demonstrando que podemos encontrar outros fenótipos celulares que não macrófagos CD68+, constituindo as vilosites. Talvez as demais células sejam células de Hofbauer que, embora sejam macrófagos, não apresentam em sua superfície marcadores para CD68.

\section{ABSTRACT}

Purpose: placental villitis has been correlated with perinatal infection, although a percentage of cases remains etiologically unknown. The present study was aimed at the systematic morphological study of placentas for imunohistochemical characterization of villitis and assessment of its possible correlation with maternal and fetal outcome.

Methods: a hundred and twenty-eight placentas were studied. Gross examination was performed and all collected fragments were analyzed microscopically by the hematoxylineosin method. Villits was classified according to the inflammatory degree in to mild, moderate and severe. The immunohistochemical study to identify infectious agents was performed using monoclonal antibodies against Toxoplasma gondii and Cytomegalovirus. For inflammatory cell phenotype identification monoclonal antibodies against CD68, CD57, $C D 3$, and CD2O were used. Statistical analysis was performed with the variables: maternal age and fetal gestational age, fetal and placental weight, and fetal and maternal outcomes. To compare the two groups we used the Mann-Whitney test and for proportions we used the $\chi^{2}$ test. The differences in the mean values between the treatment groups were considered statistically significant when $p<0.05(5 \%)$.

Results: villitis was identified in $11.7 \%$ of the cases. In $40 \%$ of the cases the children were stillborn $(p=0.003)$. One case showed positive staining for toxoplasmosis while the remaining cases were negative. Imunohistochemical staining showed CD68+ cells, PanT + cells and negative CD57 and PanB cells.

Conclusion: we concluded that the intensity of the inflammatory process in the placenta was correlated with the severity of the fetal disease. The inflammatory cells in the villitis focus were macrophages; however, we could not identify infectious agents correlated with the villitis.

KEYWORDS: Placenta. Villitis. Infection in pregnancy. Toxoplasmosis.

\section{Referências}

1. Harrington B, Glazier J, D’Souza S, Sibley C. System A amnino acid transporter activity in human placental microvillous membrane vesicles in relation to various anthropometric measurements in appropriate and small for gestational age babies. Pediatr Res 1999; 45:810-4.

2. Agapitus E, Papadopoulou C, Kavantzas N, Papoulias J, Antonaki V, Davaris P. The contribution of pathological examination of the placenta in the investigation of the causes of foetal mortality. Arch Anat Cytol Pathol 1996; 44:5-11.

3. Sheffield JS, Sanchez PJ, Wendel GD Jr, et al. Placental histopathology of congenital syphilis. Obstet Gynecol 2002; 100:126-33.

4. Mwanyumba F, Gaillard P, Inion I, et al. Placental inflammation and perinatal transmission of HIV-1. J Acquir Immune Defic Syndr 2002; 29:262-9.

5. Kaplan C. The placenta and viral infections. Semin Diagn Pathol 1993; 10:232-50.

6. Altemani AM, Bittencourt AL, Lana AM. Immunohistochemical characterization of the inflammatory infiltrate in placental Chagas' disease: a qualitative and quantitative analysis. Am J Trop Med Hyg 2000; 62:319-24. 
7. Qureshi F, Jacques SM. Maternal varicella during pregnancy: correlation of maternal history and fetal outcome with placental histopathology. Hum Pathol 1996; 27:191-5.

8. Gershon R, Strauss L. Structural changes in human placentas associated with fetal inanition or growth arrest (placental insufficiency syndrome). Am J Dis Child 1961; 102:645-6.

9. Benirschke K, Altshuler G. The future of perinatal physiopathology. In: Abranson H, editor. Symposium on the Functional Physiopathology of the Fetus and Neonate. $1^{\text {st }}$ ed. St Louis: Mosby; 1971. p. 156-68.

10.Benirschke K, Coen R, Patterson B, Key T. Villitis of known origin: varicella and toxoplasma. Placenta 1999; 20:395-9.

11.Sander CH. Hemorrhagic endovasculitis and hemorrhagic villitis of the placenta. Arch Pathol Lab Med 1980; 104:371-3.

12. Oliveira LH, Xavier CC, Lana MA. Alterações morfológicas placentárias de recém-nascidos pequenos para a idade gestacional. J Pediatr 2002; 78:397-402.

13.Altemani AM, Gonzatti AR. Vilosite de etiologia desconhecida em placentas de gestações com hipertensão arterial e de gestações com recémnascidos pequenos para a idade gestacional. Rev Assoc Med Bras 2003; 49:67-71.

14.Bjoro K Jr, Myhre E. The role of chronic non-specific inflammatory lesions of the placenta in intrauterine growth retardation. Acta Pathol Microbiol Immunol Scand [A] 1984; 92:133-7.

15.Greco MA, Wieczorek R, Sachdev R, Kaplan C, Nuovo GJ, Demopoulos RI. Phenotype of villous stromal cells in placentas with cytomegalovirus, syphillis, and non specific villitis. Am J Pathol $1992 ; 141: 835-42$.
16.Styer AK, Parker HJ, Roberts DJ, Palmer-Toy D, Toth TL, Ecker JL. Placental villitis of unclear etiology during ovum donor in vitro fertilization pregnancy. Am J Obstet Gynecol 2003; 189:1184-6.

17.Xiao J, Garcia-Lloret M, Winkler-Lowen B, Miller R, Simpson K, Guilbert LJ. ICAM-1-mediated adhesion of periphereal blood monocytes to the maternal surface of placental syncytiotrophoblasts: implications for placental vilittis. Am J Pathol 1997; 150:1845-60.

18.Doss BJ, Greene MF, Hill J, Heffner LJ, Bieber FR, Genest DR. Massive chronic intervilositis associated with recurrent abortions. Hum Pathol 1995; 26:1245-51.

19.Russell P. Inflammatory lesions of the human placenta. III. The histopathology of villitis of unknown aetiology. Placenta 1980; 1:227-44.

20.Boyd TK, Redline RW. Chronic histiocytic intervillositis: a placental lesion associated with recurrent reproductive loss. Hum Pathol 2000; 31:1389-96.

21.Nybo Andersen AM, Wohlfahrt J, Christens P, Olsen J, Melbye M. Maternal age and fetal loss: population based register linkage study. BMJ 2000; 320:1708-12.

22.Nordenvall M, Sandstedt, B. Placental villitis and intrauterine growth retardation in a Swedish population. APMIS 1990; 98:19-24.

23.Redline RW, Patterson P. Villitis of unknown etiology is associated with major infiltration of fetal tissue by maternal inflammatory cells. Am J Pathol 1993; 143:473-9.

24.Altemani AM. Immunohistochemical study of the inflammatory infiltrate in villitis of unknown etiology. A qualitative and quantitative analysis. Pathol Res Pract 1992; 188:303-9.

Recebido em: 27/9/2004 Aceito com modificações em: 17/11/2004 